\title{
Relationship between Serum Bilirubin and Carotid Atherosclerosis in Hypertensive Patients
}

\author{
Xia-Feng Yang ${ }^{1}$, Yuan-Zhi Chen ${ }^{1}$, Jiang-Li Su ${ }^{1}$, Feng-Yun Wang ${ }^{1}$ and Le-Xin Wang ${ }^{2}$
}

\begin{abstract}
Objective To investigate the relationship between serum bilirubin and carotid atherosclerosis in patients with hypertension.

Patients and Methods Carotid artery ultrasonography was performed in 198 patients (104 males, average age of $65.6 \pm 7.1$ years) with hypertension. Serum levels of bilirubin and C-reactive proteins (CRP) were measured at the same time.

Results Carotid atherosclerosis was detected in 133 patients, 87 of them had carotid artery plaque. The prevalence of stroke $(20.3 \%)$ and myocardial infarction $(13.5 \%)$ in the atherosclerosis group was higher than in the non-atherosclerosis group $(9.2 \%$ and $6.2 \%$, respectively, $\mathrm{p}<0.05)$. The average total serum bilirubin in the atherosclerosis group was lower than in the non-atherosclerosis group $(12.8 \pm 1.3 \mathrm{vs} 16.8 \pm 1.5 \mu \mathrm{mol} / \mathrm{L}, \mathrm{p}<$ $0.01)$, whereas the average serum of CRP was higher $(4.1 \pm 1.1$ vs $2.3 \pm 0.7 \mathrm{mg} / \mathrm{L}, \mathrm{p}<0.01)$. After adjusting other factors such as age, total cholesterol, diabetes and systolic blood pressure, total serum bilirubin was negatively associated with carotid atherosclerosis in women and men, with odds ratios of 0.49 (95\% CI, 0.28 to $0.71 ; \mathrm{p}<0.01)$ and $0.66(95 \% \mathrm{CI}, 0.46$ to $0.80 ; \mathrm{p}<0.01)$. Serum CRP was positively correlated to carotid atherosclerosis, with odds ratios of 1.76 (95\% CI, 1.36 to 2.04; $\mathrm{p}<0.01$ ) in women and 1.95 (95\% CI, 1.46 to 2.82; $\mathrm{p}<0.01)$ in men.

Conclusion Carotid atherosclerosis was associated with a high prevalence of stroke or myocardial infarction in hypertensive patients. Serum bilirubin was negatively associated with carotid atherosclerosis.
\end{abstract}

Key words: carotid plaque, atherosclerosis, bilirubin, C-reactive protein, hypertension

(Inter Med 48: 1595-1599, 2009)

(DOI: 10.2169/internalmedicine.48.2286)

\section{Introduction}

Previous studies have demonstrated that in hypertensive patients, peripheral arterial atherosclerosis is associated with an increased risk of myocardial infarction and stroke $(1,2)$. Advanced atherosclerosis is thought to be a consequence of chronic local and systemic inflammatory disease (3). Creactive protein (CRP) is a biomarker of inflammation, and an independent predictor for future vascular events, such as carotid atherosclerotic activity, myocardial infarction and stroke (4-6). Bilirubin is reported to possess antioxidant properties (7). In recent years, several studies have demonstrated that the total serum bilirubin level is negatively asso- ciated with carotid plaque in women and men (8-10), suggesting that bilirubin may act as a defense mechanism against atherosclerosis.

The primary purpose of this study was to investigate the association between serum bilirubin and carotid atherosclerosis in patients with hypertension. The relationship between serum CRP and carotid atherosclerosis was also investigated.

\section{Patients and Methods}

\section{Patient selection}

This study was approved by the institution review board of Liaocheng People's Hospital. Informed consent was ob-

${ }^{1}$ Department of Neurology, Liaocheng People's Hospital and Liaocheng Clinical School of Taishan Medical University, Shandong, People's Republic of China and ${ }^{2}$ School of Biomedical Sciences, Charles Sturt University, Wagga Wagga, Australia

Received for publication March 20, 2009; Accepted for publication June 2, 2009

Correspondence to Dr. Le-Xin Wang, lwang@csu.edu.au 
tained from all participants before the study. Between 2004 and 2008, 198 patients with primary hypertension were selected for this study. There were 104 males and 94 females, and an average age of 65.6 \pm 7.1 (range 46-82) years.

All patients underwent a thorough physical examination, chest radiography, ECG and echocardiography. Blood biochemistry profile was also performed. None of the patients had significant hepatic or renal dysfunction, or any other non-cardiovascular disorder.

\section{Carotid artery ultrasound examination}

Carotid artery was examined bilaterally by high-resolution B-mode ultrasonography using an ultrasonogram (Sequoia C 512; Acuson, Siemens) equipped with a 7.5-MHz transducer. Carotid arteries were examined at the levels of the common carotid, bifurcation, and internal carotid arteries from transverse and longitudinal orientations by trained sonographers. The intima-media thickness was measured using a computerassisted method by experienced sonograph technicians who were unaware of the subjects' clinical and laboratory findings. Plaque was defined as clearly isolated focal thickening of the intimal-medial layer with an intimal-medial thickness of $\geq 1.3 \mathrm{~mm}$ at the common or internal carotid arteries or the carotid bulb. Carotid intima-media wall thickening was said to occur when the intima-media thickness, which was measured at the far wall of the distal $10 \mathrm{~mm}$ of the common carotid artery, was $\geq 0.9 \mathrm{~mm}$. The presence of intima-media wall thickness or plaque was regarded as a sign of atherosclerosis of the carotid artery.

According to the results of the ultrasound examination, patients were divided into atherosclerosis and nonatherosclerosis groups. The atherosclerosis group was divided into carotid plaque and no plaque subgroups.

\section{Measurement of serum bilirubin and CRP}

Venous blood was collected following overnight fasting for at least 12 hour. Serum total bilirubin levels were measured by the bilirubin oxidase method. CRP was measured by an automatic immunonephelometer with a sensitivity of $0.02 \mathrm{mg} / \mathrm{dL}$ (Behring NA latex CRP; Behring Institute).

\section{Statistical analysis}

SPSS 11.5 software was used to perform the statistical analysis. Data were expressed as means \pm SD. Bilirubin and CRP concentrations were evaluated by 1-way ANOVA. Multivariate logistic regression analysis was used to evaluate the relations between serum bilirubin or CRP values and carotid atherosclerosis. Categorical data were analyzed by Chisquare test. $\mathrm{p}<0.05$ was considered statistically significant.

\section{Results}

\section{General findings}

As shown in Table 1, there was no significant difference in age, sex, average blood pressure or body mass index be- tween patients with or without carotid atherosclerosis. However, the total blood cholesterol and triglyceride levels in the atherosclerosis group were higher than in the nonatherosclerosis group $(\mathrm{p}<0.05)$. The prevalence of type 2 diabetes, the average fasting plasma glucose and HbA1c in the atherosclerosis group were higher than in the nonatherosclerosis group $(\mathrm{p}<0.05)$. The prevalence of stroke and myocardial infarction in the atherosclerosis group was also higher than in the non-atherosclerosis group $(\mathrm{p}<0.05)$. Furthermore, the prevalence of stroke in the carotid artery plaque group was higher than in the non-atherosclerosis group $(\mathrm{p}<0.01)$. Within the atherosclerosis group, the average levels of total cholesterol and triglycerides in patients with carotid plaques were higher than in patients without carotid plaques $(\mathrm{p}<0.05)$.

The management of hypertension was summarized in Table 2. There was no significant difference in the type of anti-hypertensive drugs used in the atherosclerosis and nonatherosclerosis groups $(p>0.05)$. The percentage of patients who did not receive any antihypertensive drugs was also similar between the two groups $(\mathrm{p}>0.05)$.

\section{Comparison of serum bilirubin and CRP between atherosclerosis and non-atherosclerosis groups}

Table 3 shows the measurement results of serum bilirubin and CRP. The direct, indirect and total serum bilirubin in the atherosclerosis group was lower than in the nonatherosclerosis group $(\mathrm{p}<0.05)$. The serum CRP level in the atherosclerosis group was higher than in the nonatherosclerosis group $(\mathrm{p}<0.05)$.

After adjusting for age, smoking status, total cholesterol, low-density lipoprotein, triglycerides, blood glucose, HbA1c, systolic blood pressure and BMI, the total serum bilirubin level was found to be negatively associated with carotid atherosclerosis in women and men, with odds ratios of 0.49 ( $95 \% \mathrm{CI}, 0.28$ to $0.71 ; \mathrm{p}<0.01)$ in women and $0.66(95 \%$ CI, 0.46 to $0.80 ; \mathrm{p}<0.01)$ in men. On the other hand, serum CRP level was found to be a risk factor for carotid atherosclerosis, with odds ratios of 1.76 (95\% CI, 1.36 to 2.04 ; p < $0.01)$ in women and 1.95 (95\% CI, 1.46 to 2.82 ; $<<0.01)$ in men.

In patients with atherosclerosis, there was no significant difference in serum total bilirubin $(13.5 \pm 1.1$ vs $13.0 \pm 1.1$ $\mu \mathrm{mol} / \mathrm{L})$ or CRP $(4.4 \pm 0.6$ vs $3.9 \pm 0.6 \mathrm{mg} / \mathrm{L})$ levels between patients with and without carotid plaques $(\mathrm{p}>0.05)$.

\section{Discussion}

Ishizaka and colleagues (8) first reported the association between the levels of serum bilirubin and the risk of atherosclerosis in 2001. They investigated carotid plaques in a heterogeneous group of 1,741 subjects during a general health check-up. They found that the 330 subjects with carotid plaque had significantly lower serum bilirubin level than those without carotid plaque. After adjustment of sex, age, status of cigarette smoking, hypertension, serum levels of to- 
Table 1. Baseline Characteristics

\begin{tabular}{|c|c|c|c|c|}
\hline & \multicolumn{3}{|c|}{ Atherosclerosis } & \multirow{2}{*}{$\begin{array}{l}\text { Non-atherosclerosis } \\
n=65\end{array}$} \\
\hline & $\begin{array}{l}\text { Plaque } \\
(n=86)\end{array}$ & $\begin{array}{l}\text { No Plaque } \\
(n=47)\end{array}$ & Total $(n=133)$ & \\
\hline Age & $65.5 \pm 7.1$ & $63.5 \pm 6.7$ & $65.7 \pm 7.1$ & $64.5 \pm 6.2$ \\
\hline Sex (M) & $52.3 \%$ & $53.2 \%$ & $52.6 \%$ & $52.3 \%$ \\
\hline $\begin{array}{l}\text { Systolic BP (mm } \\
\mathrm{Hg})\end{array}$ & $166.7 \pm 11.2$ & $164.7 \pm 13.2$ & $165.2 \pm 11.9$ & $163.2 \pm 12.2$ \\
\hline $\begin{array}{l}\text { Diastolic BP (mm } \\
\mathrm{Hg})\end{array}$ & $82.4 \pm 9.2$ & $84.4 \pm 10.7$ & $83.4 \pm 9.1$ & $83.9 \pm 10.2$ \\
\hline BMI $\left(\mathrm{kg} / \mathrm{m}^{2}\right)$ & $24.7 \pm 4.2$ & $24.6 \pm 5.7$ & $24.6 \pm 5.9$ & $24.1 \pm 5.2$ \\
\hline TCL (mmol/L) & $5.87 \pm 0.90^{*}$ & $4.86 \pm 0.68$ & $5.52 \pm 0.87$ & $4.58 \pm 0.82^{\#}$ \\
\hline $\mathrm{LDL}(\mathrm{mmol} / \mathrm{L})$ & $2.35 \pm 0.70$ & $2.18 \pm 0.64$ & $2.35 \pm 0.70$ & $2.32 \pm 0.62$ \\
\hline TG (mmol/L) & $2.36 \pm 0.61^{*}$ & $1.92 \pm 0.20^{*}$ & $2.28 \pm 0.16$ & $1.61 \pm 0.39^{\#}$ \\
\hline $\mathrm{HDL}(\mathrm{mmol} / \mathrm{L})$ & $0.96 \pm 0.26$ & $0.97 \pm 0.29$ & $0.96 \pm 0.34$ & $1.01 \pm 0.36$ \\
\hline Type 2 diabetes & $31.4 \%(27)$ & $25.5 \%(12)$ & $29.3 \%(39)$ & $15.4 \%(10)^{\#}$ \\
\hline $\begin{array}{l}\text { Fasting plasma } \\
\text { glucose }(\mathrm{mmol} / \mathrm{L})\end{array}$ & $5.9 \pm 0.6^{*}$ & $5.2 \pm 0.7$ & $5.4 \pm 0.5$ & $4.6 \pm 0.3^{\#}$ \\
\hline HbA1c (\%) & $6.7 \pm 1.9$ & $6.5 \pm 1.6$ & $6.6 \pm 1.7$ & $5.0 \pm 0.6^{*}$ \\
\hline Stroke & $25.6 \%(22)$ & $10.6 \%(5)$ & $20.3 \%(27)$ & $9.2 \%(6)^{\#}$ \\
\hline MI & $15.0 \%(13)$ & $16.1 \%(13)$ & $13.5 \%(18)$ & $6.2 \%(4)^{\#}$ \\
\hline
\end{tabular}

TCL: total cholesterol. LDL: low-density lipoprotein. MI: myocardial infarction. BP: blood pressure. $* \mathrm{p}<0.05$ compared with the no-plaque group; $\# \mathrm{p}<0.05$ compared with the total of the atherosclerosis group.

Table 2. Management of Hypertension

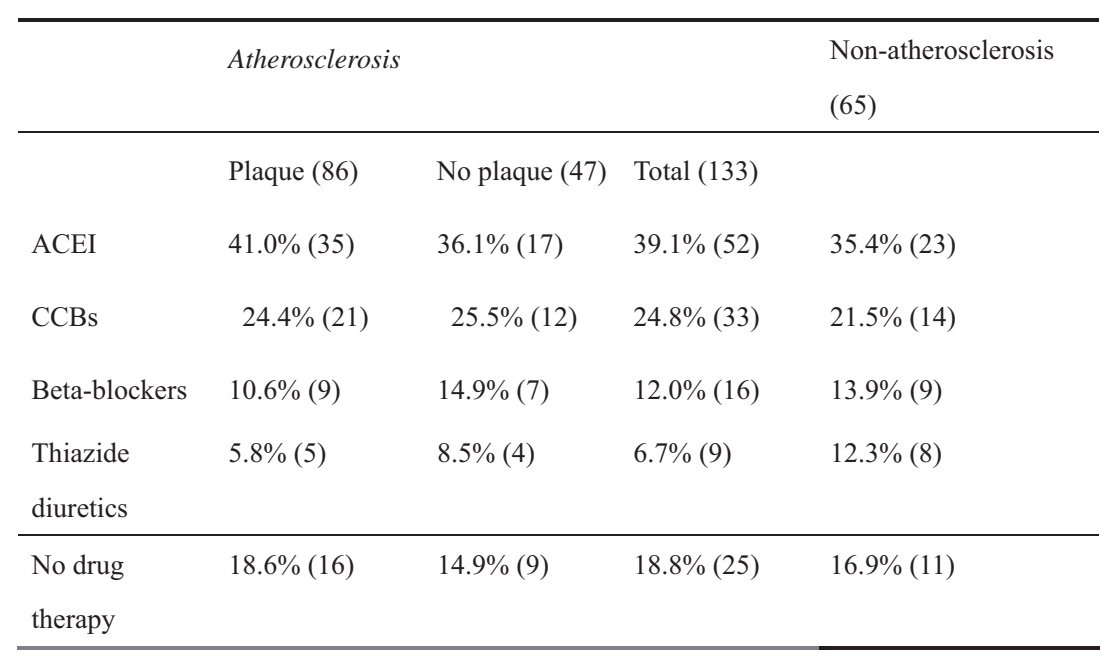

ACEI: angiotensin-converting enzyme inhibitor; CCBs: calcium channel blockers.

tal cholesterol, HDL cholesterol, triglyceride and HbA1c, lated with the risk of carotid plaque (8). The same group of the serum bilirubin concentration was still inversely corre- investigators later conducted a larger correlation study on 
Table 3. Serum Bilirubin and CRP Results

\begin{tabular}{l|lll}
\hline & $\begin{array}{l}\text { Atherosclerosis } \\
(\mathrm{n}=133)\end{array}$ & $\begin{array}{l}\text { Non-atherosclerosis } \\
(\mathrm{n}=65)\end{array}$ & $\mathrm{p}$ \\
\hline $\begin{array}{l}\text { Direct bilirubin } \\
(\mu \mathrm{mol} / \mathrm{L})\end{array}$ & $5.3 \pm 0.7$ & $7.9 \pm 0.7$ & $<0.05$ \\
$\begin{array}{l}\text { Indirect bilirubin } \\
(\mu \mathrm{mol} / \mathrm{L})\end{array}$ & $7.9 \pm 0.9$ & $9.4 \pm 1.0$ & $<0.05$ \\
$\begin{array}{l}\text { Total bilirubin } \\
(\mu \mathrm{mol} / \mathrm{L})\end{array}$ & $12.8 \pm 1.3$ & $16.8 \pm 1.5$ & $<0.05$ \\
$\mathrm{CRP}(\mathrm{mg} / \mathrm{L})$ & $4.1 \pm 1.1$ & $2.3 \pm 0.7$ & $<0.05$ \\
\hline
\end{tabular}

8,144 subjects who underwent a general health check-up (9). They again found that the total bilirubin level was negatively associated with carotid plaque in men and women (9). Furthermore, the total bilirubin level was inversely correlated with metabolic syndrome (9). Recently, Erdogan et al (10) studied the relationship between serum bilirubin and carotid intima-media thickness in 91 healthy subjects. They reported that carotid intima-media thickness was significantly greater in both women and men with lower serum bilirubin concentrations compared to the subjects with elevated serum bilirubin concentrations (10). In the same study, in men or women with lower serum bilirubin concentrations flow-mediated dilatation, an indicator of vascular endothelial function, was significantly lower than in those with higher serum bilirubin concentrations (10).

Our study focused on a group of hypertensive patients who had no other illnesses. Patients with carotid atherosclerosis had a lower level of serum bilirubin and a higher level of CRP than patients without carotid atherosclerosis. There was a clear inverse correlation between carotid atherosclerosis and serum bilirubin. The results from our hypertensive patients are consistent with those from the healthy subjects (8-10). Increased intima-media thickness and plaque formation in the carotid artery are markers of atherosclerosis. Both conditions are related to aging and are accelerated by hypertension and other cardiovascular risk factors (11). Plaque measurement showed a better correlation with atherosclerotic events than intima-media thickness (12). In the present study, patients with carotid plaques had higher levels of total serum cholesterol and triglycerides, but the serum bilirubin or CRP levels were similar between the two groups. These results suggest that bilirubin and CRP are related to atherosclerosis but not necessarily to the pathogenesis of carotid plaques.

Although a causal or resultant relationship between serum bilirubin and carotid atherosclerosis is yet to be established, there is some indirect evidence which suggests that bilirubin may be anti-atherosclerotic. Previous studies have shown that bilirubin exerts an antioxidant effect $(2,13)$. Antioxidative agents are known to act against the formation of atheromatous lesions in animal models and in humans $(14,15)$. A recent cross-section survey revealed that a $1.7 \mathrm{micromol} / \mathrm{L}$ increment in bilirubin level was associated with a $9 \%$ reduced odds of stroke and with a $10 \%$ reduced odds of an adverse stroke outcome, suggesting that bilirubin may serve to protect from atherosclerotic stroke events and from neurologic damage in stroke (2).

In conclusion, this study demonstrated that in hypertensive patients, carotid atherosclerosis is associated with an increased prevalence of stroke and myocardial infarction. The average levels of serum bilirubin in patients with carotid atherosclerosis were lower than in patients without carotid atherosclerosis. The inverse correlation between the serum bilirubin level and carotid atherosclerosis suggests that bilirubin may be anti-atherosclerotic through its antioxidant action.

\section{References}

1. Makin A, Lip GY, Silvennan S, Beevers DG. Peripheral vascular disease and hypertension: a forgotten association. J Hum Hypertens 15: 447-454, 2001.

2. Perlstein TS, Pande RL, Creager MA, Weuve J, Beckman JA. Serum total bilirubin level, prevalent stroke, and stroke outcomes: NHANES 1999-2004. Am J Med 121: 781-788, 2008.

3. Libby P. Inflammation in atherosclerosis. Nature 420: $868-874$, 2002.

4. Hashimoto H, Kitagawa K, Hougaku H, et al. C-reactive protein is an independent predictor of the rate of increase in early carotid atherosclerosis. Circulation 104: 63-67, 2001.

5. Ridker PM. Role of inflammatory biomarkers in prediction of coronary heart disease. Lancet 358: 946-948, 2001.

6. Elias-Smale SE, Kardys I, Oudkerk M, Hofman A, Witteman JC. $\mathrm{C}$-reactive protein is related to extent and progression of coronary and extra-coronary atherosclerosis; results from the Rotterdam study. Atherosclerosis 195: e195-e202, 2007.

7. Stocker R, Yamamoto Y, McDonagh AF, Glazer AN, Ames BN. Bilirubin is an antioxidant of possible physiological importance. Science 235: 1043-1046, 1987.

8. Ishizaka N, Ishizaka Y, Takahashi E, Yamakado M, Hashimoto H. High serum bilirubin level is inversely associated with the presence of carotid plaque. Stroke 32: 580-583, 2001.

9. Ishizaka N, Ishizaka Y, Toda E, Nagai R, Yamakado M. Association between serum uric acid, metabolic syndrome, and carotid atherosclerosis in Japanese individuals. Arterioscler Thromb Vasc Biol 25: 1038-1044, 2005.

10. Erdogan D, Gullu H, Tok D, et al. Low serum bilirubin levels are independently and inversely related to impaired flow-mediated vasodilation and increase carotid intima-media thickness in both 
men and women. Athersclerosis 184: 431-439, 2006.

11. Bonithon-Kopp C, Touboul PJ, Berr C, et al. Relation of intimamedia thickness to atherosclerotic plaques in carotid arteries: the vascular aging (EVA) study. Arterioscler Thromb Vasc Biol 16: 310-316, 1996.

12. Ebrahim S, Papacosta O, Whincup P, et al. Carotid plaque, intima media thickness, cardiovascular risk factors, and prevalent cardiovascular disease in men and women: the British Regional Heart Study. Stroke 30: 841-850, 1999.

13. Wu TW, Fung KP, Wu J, Yang CC, Weisel RD. Antioxidation of human low density lipoprotein by unconjugated and conjugated bilirubins. Biochem Pharmacol 51: 859-862, 1996.

14. Crawford RS, Kirk EA, Rosenfeld ME, LeBoeuf RC, Chait A. Dietary antioxidants inhibit development of fatty streak lesions in the LDL receptor-deficient mouse. Arterioscler Thromb Vasc Biol 18: 1506-1513, 1998.

15. Azen SP, Qian D, Mack WJ, et al. Effect of supplementary antioxidant vitamin intake on carotid arterial wall intima-media thickness in a controlled clinical trial of cholesterol lowering. Circulation 94: 2369-2372, 1996.

\section{(C) 2009 The Japanese Society of Internal Medicine http://www.naika.or.jp/imindex.html}

